\title{
Usable Web-based Calendaring for Blind Users
}

\author{
Brian Wentz \\ Frostburg State University \\ Frostburg, MD, USA \\ bwentz@acm.org
}

\author{
Jonathan Lazar \\ Towson University \\ Towson, MD, USA \\ jlazar@towson.edu
}

\begin{abstract}
While a calendar is generally thought of as a visual representation of a day, week, month or year; in its simplest form, a calendar consists of scheduled events with associated dates, times, locations and descriptions. In this paper we describe the design and evaluation of a web-based calendar prototype for blind individuals who use screen readers to access their computers and the Internet. With our new web-based calendar prototype, the successful completion across common calendar tasks improved from a mean of $\mathbf{4 3}$ per cent across other web-based email calendars to a mean of 96 per cent in our prototype calendar. This empirical study illustrates the types of simple design changes that can dramatically improve the level of access and usability of dynamic, web-based calendar interfaces for blind users.
\end{abstract}

Blind, visual impairment, calendar, screen reader, usability, human-computer interaction.

\section{INTRODUCTION}

With the increasing importance of collaborative applications in the workplace, the ability for all users to be able to fully use those applications is critical. Unemployment is a major problem for people with disabilities, and employment requires the effective use of workplace software applications, such as email, intranets, chat tools, and shared calendars. If a workplace software tool is technically inaccessible, this may have the unintended effect of social exclusion from certain workplace activities, and may lower productivity. For instance, how would blind users be perceived by colleagues, if they cannot do group scheduling, using the same web-based calendar that all of the other employees use?

Previous usability testing (Wentz B. And Lazar, J. 2011) has established that, for blind users, webbased email applications are more likely to have usability problems than stand-alone, desktop email applications, and this research also revealed that extended email features such as calendaring exhibit the lowest levels of usability for blind users (a task success rate of only 43 per cent for calendar tasks across all four of the web-based email applications as tested by 15 blind users). Each user tested one web-based email application, so each application was evaluated by three to four users in the previous research study. Desktop email applications (such as those from Microsoft and Mozilla) were also evaluated during the previous study. The web-based applications tested in that study were Gmail, Outlook Web Access 2007 Light, Yahoo Mail Classic, and Windows Live (Hotmail). For the comparisons made later on in this paper, we will only consider the data from the web-based applications in our previous study, due to the fact that the prototype calendar is webbased. Based on our previous data, it was determined that the development of a web-based calendar, usable by blind users, could illustrate the types of improvements that could be implemented on web-based email interfaces.

\section{CREATING A USABLE CALENDAR}

There have been many suggestions for making calendars more accessible on the Web (McAvoy, M. 2007; Pilgrim, M. 2002), but most of these suggestions relate to static calendar content presented on web sites. One suggestion of how to approach the design of an accessible, interactive calendar is to linearize the data from the visual perspective of most calendars and allow the user to search for a period of dates on a calendar in order to focus on the dates of interest to the user (Millard, B. 2006).

The creation of a web-based calendar designed specifically with usability for a screen reader in mind necessitated that the traditional or most common software-based representations of calendars be momentarily ignored. Instead, this 
calendar design was approached from the perspective of the calendar components merely being stored data, and the calendar interface itself was treated as merely a point of access to that data.

\subsection{Related research and products}

There are few well-known calendars that have been specifically designed with the screen reader user in mind, but one such example is the WebblE calendar (King, A. 2010). It is a simple, desktopbased calendar application which illustrates the ease of use that a calendar can have, because it has simple buttons and menus that can easily be navigated by a keyboard and screen reader. It is, however, missing things like event location, the ability to add additional information, and confirmations of events that are deleted. While the WebblE calendar is not a web-based application, it does serve as an example of a usable desktopbased implementation of a calendar. Figure 1 shows a screenshot of the WebblE calendar interface.

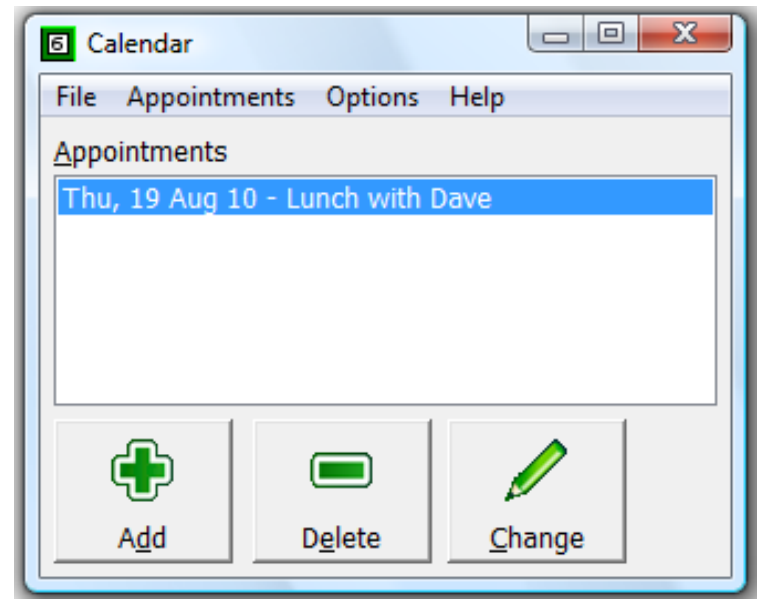

Figure 1: Screenshot of the WebblE calendar

WAI-ARIA is a planned W3C standard to outline how developers can make dynamic content more accessible, particularly for those who are blind and use a screen reader or for those with motor impairment who cannot use pointing devices such as a mouse. Some developers and companies, such as Google, have already attempted to change the design of some of their applications (such as email calendars) to address the proposed recommendations for dynamic content. The future possibilities for WAI-ARIA are encouraging; however, the current benefits of the changes related to WAI-ARIA are often limited to users of a particular web browser (Google 2010b). While design should not exclude the benefits for WAIARIA, good design should also account for users whose software versions may not fully support WAI-ARIA.
A recent research study at the University of Manchester (UK) also focused on the problem of audio access to calendars (Brown, A., Jay, C., and Harper, S. 2010). Researchers designed and tested a non-visual interface for selecting dates on web-based forms. The goal was to provide audio access to date selection while automating the formatting of those dates and avoiding errors. Researchers noted that the calendar date entry system reduced a common confusion (regarding how to format a date) that can occur when a user is asked to manually type a date.

\subsection{The calendar design}

In order to illustrate that the previously tested email calendar interfaces could be easily modified to be easier to use for blind users, the API for one of the email applications was used. For this purpose, the Google Calendar was chosen, due to its poor performance in our previous usability testing (25 per cent success rate on the calendaring tasks based on evaluation with four users) (Wentz, B. and Lazar, J. 2011), the growing commercial and academic use of Google for email, and the fact that nine out of 15 participants in the previous usability testing reported having used Gmail (with only two of those no longer using it) (ibid). Google publicly posts the API for its calendar, so this information was used when designing the calendar interface (Google 2010a). The calendar interface was designed using PHP and the Zend library (which is necessary to connect to the Google API).

To design a usable web-based calendar, the data on email calendars from the previous stage of our research (Wentz, B. and Lazar, J. 2011) was used as the basis for designing a calendar that would be usable by a screen reader and keyboard. These techniques included:

- Designing all navigation for keyboard use;

- Using appropriate HTML headings and links to assist with navigation;

- Providing feedback to the users for things such as which date is the current focus and clear confirmations for any actions that are taken;

- Providing a method of viewing events that is not reliant on the visual view of a calendar "month."

As a result, all calendar pages were designed to clearly indicate the current date and the date of focus for each event (as illustrated in Figure 2).

Navigation was designed to use hyperlinks and text fields, the ability to search was incorporated into each calendar page, and clear confirmation was included for any action taken. Also, a choice of returning to the main menu is given after each 
action is taken. Headings were used for event titles since they can be easily accessed through screen readers, and the tab order was verified for logical order.

Today's date is Wednesday June 092010

\section{Viewing calendar events:}

1 event(s) found.

\section{Event Title: Meeting with Tom Michaels}

Location: New York Office

Starts: Wednesday June 162010 9:00 AM

Ends: Wednesday June 162010 11:30 AM

Additional information: bring portfolio

edit this event I delete this event

Add a New Event

Search for Events by title or description

\section{Search}

Return to Main Menu

Figure 2: Our calendar interface: clear calendar events, with the current date and focus evident through the screen reader

Links on the main calendar page include adding an event, going to today's, yesterday's, or tomorrow's events, going to a particular date, viewing the next seven days, viewing the next 30 days, viewing all future events or viewing all past and future events (as shown in Figure 3). These types of links were used to simplify navigation. Also, to avoid errors when dates and times are entered, the prototype interface clearly identifies the format of the input which is being requested.

\begin{tabular}{l} 
Today's date is Wednesday July 212010 \\
Calendar Menu \\
Godd a New Event \\
Go to Today \\
Go to Yesterday \\
Ge tomorrow \\
View Next Seven Days \\
Go to a Particular Date \\
View all Future Events \\
View all Past and Future Events \\
\hline
\end{tabular}

Figure 3: Calendar menu on our prototype design

\subsection{Pilot study and modifications}

In early June, 2010, pilot usability testing of the web-based calendar interface was conducted with two blind users. Confirmation for actions taken on the calendar was changed to include the title of the event impacted (instead of "event deleted successfully", the specifics of what was deleted were included). Some field labels were clarified, such as on the "Add Event" page (where the field label read simply "enter the month" it was changed to "enter a two digit starting month" to avoid confusion and errors). Pilot study participants noted that the interface was straightforward and easy to use, even though both pilot test participants had never before used an email calendar due to having heard about the difficulty from others.

\subsection{Evaluation process}

For the purposes of our study, the participants were self-reported as blind with no residual vision, at least 18 years of age, and screen reader users not able to use screen magnification. The mean age of the 15 participants was 38.7 , ranging in age from 22 to 60 . Of the 15 participants, 14 were college graduates or had completed some college, and 12 of the 15 participants were female.

Each participant was asked preliminary questions regarding their experience with email calendars, during which 60 per cent of the 15 participants noted previous experience with email calendars, but the desktop-based Outlook calendar (various versions) was the only calendar which participants reported currently using.

Each participant was then asked to complete the same three calendar-related tasks that were used for our prior email usability testing (Wentz, B. and Lazar, J. 2011) as illustrated in Table 1.

Table 1: Calendar-related tasks

\begin{tabular}{|l|l|}
\hline \multicolumn{1}{|c|}{ Task } & \multicolumn{1}{c|}{ Description } \\
\hline 1 & Viewing an appointment \\
\hline 2 & Creating an appointment \\
\hline 3 & Deleting an appointment \\
\hline
\end{tabular}

JAWS 10 and Internet Explorer 8 were used on a pre-configured netbook with an external keyboard and speakers, and fictitious calendar events were placed on the calendar for testing purposes. Previous research has illustrated the value of assessing web-based interfaces with a screen reader rather than assessment through automated processes (Mankoff, J., Fait, H., and Tran, T. 2005).

\section{RESULTS}

The comparative results of the evaluation were dramatic. Task one on the prototype (finding and viewing a calendar appointment) showed a mean completion rate of 100 per cent versus the 63 per 
cent completion rate exhibited across web-based calendars examined in the previous study (Wentz, B. and Lazar, J. 2011) and the 50 per cent completion rate shown by the standard Google Calendar interface (two out of four users). It is important to note that the following comparisons to our previous study (ibid) are focused specifically on the four web-based applications that we evaluated (Google, Outlook Web Access 2007 Light, Windows Live, and Yahoo Mail Classic) rather than all seven applications (which would have included desktop-based applications). Task one on the prototype took a mean time of 63.2 seconds for successfully completed tasks. Across web-based calendars examined in the previous study, the mean time for successfully completed tasks was 278.7 seconds, and there was a mean time of 168 seconds for the Google Calendar (ibid).

Task two (creating a calendar appointment) had an 87 per cent completion rate versus the 33 per cent for all web-based calendars in the previous study and the 25 per cent completion rate shown by the Google Calendar in the previous study (one out of four users) (ibid). Task two on the prototype took a mean time of 203.7 seconds for successfully completed tasks, compared to the 185.1 seconds mean across web-based calendars and the 102 seconds taken by a single successful Google Calendar user during the evaluation of task two during the previous study (ibid).

Task three (deleting a calendar appointment) showed a 100 per cent completion rate for the webbased prototype versus the 33 per cent and 0 per cent completion rates shown with all web-based calendars and the Google Calendar interface, respectively, in the previous study (ibid). Task three on the prototype took a mean time of 130.5 seconds for successfully completed tasks compared to the 202.1 seconds mean across webbased calendars. No users were able to successfully complete task three in the Google Calendar during the previous study (ibid). For complete data on the comparative performance, review Table 2.

Comments from the users after testing the prototype interface included statements such as "this would be feasible for work purposes," "this would be a good and usable tool," "I would use a calendar if it were this easy," "very simple because of the clear headings and links," "tell me where I can get this," and "this is the most usable calendar that I have ever used." Two users did note that drop-down date selection would have been preferred rather than text fields for event data entry.
Table 2: Comparing the mean completion rate and time (in seconds) for successful tasks across the web-based, Google and prototype calendars

\begin{tabular}{|l|l|l|l|}
\hline Task & $\begin{array}{c}\text { Web-based } \\
\text { Calendars } \\
\text { (Wentz, B. } \\
\text { and Lazar, J. } \\
\text { 2011) }\end{array}$ & $\begin{array}{c}\text { Google } \\
\text { Calendar } \\
\text { (Wentz, B. } \\
\text { and Lazar, } \\
\text { J. 2011) }\end{array}$ & $\begin{array}{c}\text { New } \\
\text { Prototype } \\
\text { Calendar }\end{array}$ \\
\hline 1 & $63 \% / 278.7$ & $50 \% / 168$ & $100 \% / 63.2$ \\
\hline 2 & $33 \% / 185.1$ & $25 \% / 102$ & $87 \% / 203.7$ \\
\hline 3 & $33 \% / 202.1$ & $0 \% / N / A$ & $100 \% / 130.5$ \\
\hline Mean & $43 \% / 222$ & $25 \% / 135$ & $96 \% / 132.5$ \\
\hline
\end{tabular}

\section{DISCUSSION}

While the interface and the changes to the "traditional" calendar approach may at first seem simplistic, this prototype and the usability testing illustrate the types of simple improvements (many of which are based on WCAG 2.0 recommendations) that can be made or added to an interface to improve the usability of dynamic, web-based applications. The goal of our research was not to create a separate interface but to illustrate that dynamic web content (like calendars) can be accessible. This could perhaps be addressed as a new user-selected view for existing calendars. Specific changes we addressed in our prototype design are as follows:

1. Users can navigate the calendar interface with the keyboard alone.

2. Functionality is not dependent on a particular browser or version.

3. There are clear confirmations of actions taken by the user.

4. Navigation of calendar events is simplified.

5. Input fields are clearly labeled to avoid input errors.

With the increasing move toward web-based applications, the usability of these interfaces is important. Changes like those discussed are relatively simple and inexpensive for developers to implement. It is not enough to simply address access and usability by designing only for new and emerging solutions which may not be available for every common web browser version and not fully functional in older screen reader versions.

\section{CONCLUSION}

In this work we have illustrated the design and usability testing of an accessible and usable interface for a dynamic web-based calendar. This type of interface and usability testing for web-based calendars has not been illustrated in prior research, and this contribution should demonstrate to 
developers and researchers alike the capabilities of inclusive interface design, regardless of the dynamic nature of a web-based application. Organizations and government entities using calendars and other dynamic, web-based application interfaces for collaborative work should be aware of the importance of all their employees and users being able to collaborate equally.

\section{REFERENCES}

McAvoy, M. (2007) Accessible Calendars for Blogs and Websites. http://georgiatechcatea.wordpress. com/2007/01/16/accessible-calendars-for-blogsand-websites/ (15 July 2010).

Brown, A., Jay, C. and Harper S. Audio access to calendars. 7th International Cross-Disciplinary Conference on Web Accessibility, Raleigh, North Carolina, (26-27 April 2010).

Millard, B. (2006). Developing Johan's Accessible Calendar. http:/sitesurgeon.co.uk/!dev/ calendar/calendar.html (20 June 2010).
Pilgrim, M. (2002). Dive Into Accessibility: 30 days to a more accessible web site.

http://diveintoaccessibility.org/day_18_giving_your_ calendar_a_real_caption.html (22 July 2010).

Google (2010). Google Calendar APIs and Tools. http://code.google.com/apis/calendar/ (20 March 2010).

Mankoff, J., Fait, H., and Tran, T. (2005). Is your web page accessible?: A comparative study of methods for assessing web page accessibility for the blind. Proceedings of the ACM Conference on Human Factors in Computing Systems, 41-50.

Google (2010). Using Google Calendar with screen readers - Google Calendar Help. http://www.google.com/support/calendar/bin/answe r.py?h1=en\&answer=152654 (21 July 2010).

King, A. WebblE, the free browser for blind people with little or no sight. http://www.webbie.org. uk/index.htm (21 July 2010).

Wentz, B. and Lazar, J. (2011). Usability Evaluation of Email Applications by Blind Users. Journal of Usability Studies. Vol. 6: No 2, 75-89. 